\title{
$\infty \sqrt{1}$ Stroke and \\ Cerebral misery perfusion due to carotid occlusive disease
}

To cite: Maddula M, Sprigg N, Bath PM, et al. Cerebral misery perfusion due to carotid occlusive disease. Stroke and Vascular Neurology 2017;2: e000067. doi:10.1136/svn2017-000067

Received 6 January 2017 Revised 9 March 2017 Accepted 13 March 2017 Published Online First 18 April 2017

\section{CrossMark}

${ }^{1}$ Acute Stroke Unit, Tauranga Hospital, Tauranga, New Zealand ${ }^{2}$ Division of Clinical

Neuroscience, University of Nottingham, Nottingham, UK ${ }^{3}$ Department of Stroke, Nottingham University Hospitals NHS Trust, Nottingham, UK

Correspondence to Dr Mohana Maddula; mohana.maddula1981@gmail. com

\author{
Mohana Maddula, ${ }^{1}$ Nikola Sprigg, ${ }^{2}$ Philip M Bath, ${ }^{2}$ Sunil Munshi ${ }^{3}$
}

\section{ABSTRACT}

Purpose Cerebral misery perfusion (CMP) is a condition where cerebral autoregulatory capacity is exhausted, and cerebral blood supply in insufficient to meet metabolic demand. We present an educational review of this important condition, which has a range of clinical manifestations.

Method A non-systematic review of published literature was undertaken on CMP and major cerebral artery occlusive disease, using Pubmed and Sciencedirect.

Findings Patients with CMP may present with strokes in watershed territories, collapses and transient ischaemic attacks or episodic movements associated with an orthostatic component. While positron emission tomography is the gold standard investigation for misery perfusion, advanced MRI is being increasingly used as an alternative investigation modality. The presence of CMP increases the risk of strokes. In addition to the devastating effect of stroke, there is accumulating evidence of impaired cognition and quality of life with carotid occlusive disease (COD) and misery perfusion. The evidence for revascularisation in the setting of complete carotid occlusion is weak. Medical management constitutes careful blood pressure management while addressing other vascular risk factors.

Discussion The evidence for the management of patients with COD and CMP is discussed, together with recommendations based on our local experience. In this review, we focus on misery perfusion due to COD. Conclusion Patients with CMP and COD may present with a wide-ranging clinical phenotype and therefore to many specialties. Early identification of patients with misery perfusion may allow appropriate management and focus on strategies to maintain or improve cerebral blood flow, while avoiding potentially harmful treatment.

\section{BACKGROUND, PATHOGENESIS AND EPIDEMIOLOGY}

Cerebral misery perfusion (CMP) is a condition where regional cerebral blood flow $(\mathrm{rCBF})$ is reduced relative to the regional metabolic demand for oxygen, and is defined by increased tissue oxygen extraction fraction $(\mathrm{OEF})$ on positron emission tomography (PET).

Atherosclerosis of the internal carotid artery or the middle cerebral artery causing occlusive disease is the most common cause of CMP. Less common causes include dissection, cardiac embolism, radiation vasculopathy,

\section{Key Points}

Consider the diagnosis of CMP in patients with:

> orthostatic TIA symptoms

- episodic limb-shaking

- watershed territory infarcts

- high-grade carotid steno-occlusive disease

- decrease in CBF with increased oxygen extraction distal to an occlusion

- Confirm diagnosis with imaging of carotids and PET/ MRI ASL

- Blood pressure should be lowered cautiously

- Consider revascularisation in selected individuals

Address episodes of hypotension aggressively

vasculitis and other chronic inflammatory conditions such as Takayasu's aortoarteritis. The occlusive process leads to reduced CBF in the distal arterial system, leading to haemodynamic impairment with disease progression. Table 1 illustrates the stages of cerebral haemodynamic impairment and the pathophysiology behind the development of CMP. Most of our understanding of CMP comes from studies looking at carotid occlusive disease (CAO), on which this review will focus.

Many patients with CAO remain asymptomatic and therefore the true prevalence of asymptomatic CAO is unknown. When 'symptomatic' (transient ischaemic attack (TIA) or stroke), the prevalence of CAO has been reported to be 6/100000; however, the authors in the study felt that true prevalence was probably higher as many patients with transient symptoms may not have sought medical help. ${ }^{1}$ In addition to the above, the difficulty in ascertaining the epidemiology of CMP is compounded by the limited availability of diagnostic investigations such as PET, and the under-recognition of symptoms that may be attributed to CMP in day-to-day clinical practise.

\section{CLINICAL AND RADIOLOGICAL FEATURES}

People with CMP may present with a range of clinical manifestations such as collapses, strokes in watershed territories or with 
Table 1 Pathophysiology of cerebral misery perfusion and stages of cerebral haemodynamic impairment

\section{Stage 1 \\ (cerebrovascular autoregulation)}

\author{
Stage 2 \\ (misery perfusion)
}

Stage 3

(end-organ compromise)
Any fall in regional cerebral perfusion pressure ( $\mathrm{rCPP})$ is matched by a fall in regional cerebrovascular resistance ( $\mathrm{rCBR}$ ) in order to maintain regional cerebral blood flow (rCBF). This is accommodated by vasodilatation and an attendant increase in regional cerebral blood volume (rCBV). Oxygen extraction factor (OEF) remains constant.

The capacity for compensatory vasodilatation is exceeded (rCVR becomes a constant) and rCBF therefore drops in tandem with rCPP. To meet their metabolic demands, neurons must 'extract more oxygen' from the passing blood-OEF increases.

If rCBF continues to fall to the extent that the brain can no longer compensate by increases in OEF, end-organ dysfunction occurs (transient ischaemic attack). If this situation persists, permanent end-organ damage (stroke) occurs.

Source: Reproduced from Gordon et $a^{40}$ with permission.

reversible neurological deficits resembling TIAs. The recognition of this condition enables appropriate management, with avoidance of unnecessary and potentially harmful treatment. Several factors such as collateral blood supply, extent of haemodynamic impairment, contribution of posterior communicating arteries and rapidity of development of CAO determine the clinical phenotype (table 2). Chronic occlusions from progressive atherosclerotic disease usually develop slowly and may allow for the formation of collaterals. Such occlusions are usually asymptomatic and are often discovered incidentally on vascular imaging after a TIA or stroke. van Laar et al found that in functionally independent patients with symptomatic CAO, the ipsilateral middle cerebral and anterior cerebral arteries were supplied by the vertebrobasilar and contralateral internal carotid arteries, respectively. ${ }^{2}$

While strokes due to $\mathrm{CAO}$ may present with clinical features similar to those due to any other aetiology, certain distinctive radiological features suggest an underlying haemodynamic mechanism. Rosary-like infarction of the centrum semi-ovale (indicating internal watershed territory infarction) has been shown to be the most sensitive marker for haemodynamic failure (figure $1 \mathrm{~A}, \mathrm{~B}$ and $\mathrm{C}) .^{3}$

In patients with transient symptoms, a detailed history may reveal an underlying haemodynamic problem.
Particular attention should be paid to symptoms provoked by standing up, exercise, postprandial or the recent commencement of antihypertensives. Rarely, patients present with transient repetitive involuntary movements in the limbs contralateral to the occluded internal carotid artery (ICA), called limb-shaking TIAs. ${ }^{4}$

Syncope and collapses may be caused by $\mathrm{CMP}{ }^{5}$ In our own experience, syncope was the presenting feature in 7 patients out of a case series of 204 patients, who were later found to have unilateral or bilateral CAO at Nottingham City Hospital. This was felt to have been an underestimation as many more patients had given a history of 'collapses', which in retrospect were probably syncopal episodes. In the same case series, nine patients presented with transient global amnesia; it is possible that the resultant cerebral ischaemia from CAO may be involved in the pathogenesis of this poorly understood condition.

In addition to the increased OEF measured by PET, which has limited availability, there may be other ways to identify those patients with CAO, haemodynamic insufficiency and a higher stroke risk. Leptomeningeal and ophthalmic collateral circulation, watershed infarction, impaired vasoreactivity to acetazolamide and orthostatic limb-shaking were all noted to predict haemodynamic failure, increased OEF on PET and higher stroke risk. ${ }^{7}$ Another study identified age, cerebral (rather than

Table 2 Clinical spectrum of carotid occlusive disease

\section{Clinical presentation}

Ischaemic stroke

Transient ischaemic attack

Limb-jerking

Ocular symptoms

Syncope

Cognitive impairment

Transient global amnesia

\section{Features}

Especially 'borderzone' infarcts in anterior or posterior cortical watershed or in the deep internal watershed territories

Can be provoked by standing posture or new antihypertensive treatment

Transient repetitive involuntary limb movements, which may be misdiagnosed as partial seizures (but with no Jacksonian march, no epileptiform activity on EEG and no improvement with anticonvulsants) ${ }^{4}$

Amaurosis fugax (complete loss of vision), or even unilateral blurred vision when looking at bright light or going from cold to warm surroundings (retinal claudication) ${ }^{41} 42$

Associated with standing posture but often with no drop in systemic blood pressure 5

Gradual deterioration in memory and other cognitive facets ${ }^{43}$

Short-term loss of memory function with no other signs of impaired cognition ${ }^{6}$ 


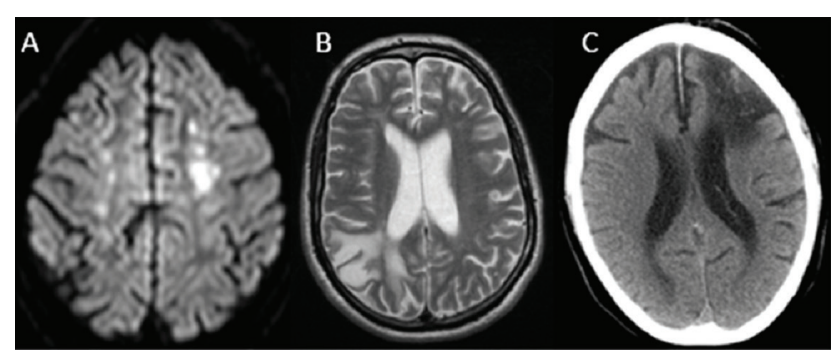

Figure 1 (A) Diffusion-weighted MRI showing internal watershed infarcts. (B) T2-weighted MRI showing posterior cortical watershed infarction. (C) CT brain showing anterior cortical watershed infarct.

retinal) symptoms, limb-shaking TIAs at presentation, history of previous strokes and the presence of leptomeningeal collaterals as risk factors for recurrent stroke in patients with symptomatic CAO. ${ }^{8}$

\section{INVESTIGATIONS}

Doppler ultrasound (DU) is the usual initial imaging modality to look for disease of the extracranial ICA. It has the benefit of being cheap and non-invasive, with a diagnostic accuracy of $97 \%$ in identifying complete CAO. ${ }^{9}$ Distinguishing between complete and near-occlusion can however be difficult: an important distinction to make, as revascularisation should be considered in patients with near occlusion. DU with the use of contrast improves the ability to distinguish between total and near-occlusion. ${ }^{10}$ It is however operator dependent, and has a limited field of view that renders the evaluation of vessel course and tandem lesions difficult.

The 'gold standard' for the evaluation of CAO is digital subtraction angiography. But it is invasive and carries the risk of neurological complications. Multislice CT angiography (figure 2A) has excellent correlation with catheter angiography in diagnosing complete versus near-occlusion of the ICA, and if used in combination with DU averts the need for catheter angiography. ${ }^{11}$ Time-of-flight

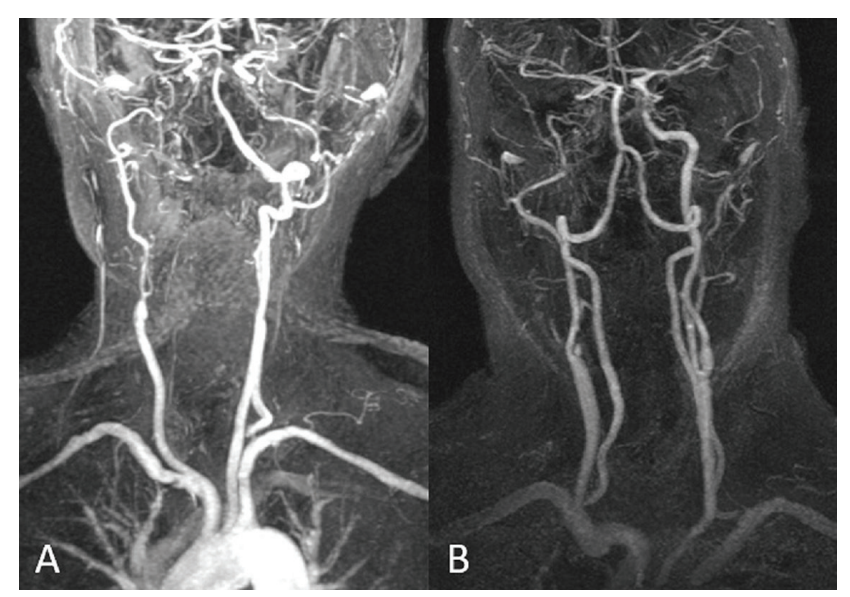

Figure 2 (A) CT angiogram showing bilateral internal carotid occlusive disease (CAO). (B) MR angiogram showing right internal CAO. magnetic resonance (MR) angiogram (figure 2B) uses the movement of blood as endogenous contrast to measure the degree of stenosis. However, it is flow-dependent, and may overestimate the degree of carotid stenosis due to signal loss in areas of slow flow from severe steno-occlusive disease. Gadolinium-enhanced MR angiogram is less susceptible to this, and has equivalent sensitivity to DU in diagnosing complete CAO, and is probably better than DU at identifying near-occlusion. ${ }^{13}$

The assessment of $\mathrm{CBF}$ is important when clinical and radiological features suggest haemodynamic impairment. PET remains the gold standard for assessing cerebral haemodynamics, but there are issues with high cost and concerns over methodology. ${ }^{14}$ Transcranial Doppler (TCD) US, Xenon-CT and single-photon emission CT have all been used to assess cerebral haemodynamic status and vasoreactivity. ${ }^{15-17}$ However, TCD US is also operator-dependent and may not technically possible in some individuals, while CT-based techniques carry radiation exposure. One advantage with TCD is the assessment of collateralisation, using the direction of flow in the anterior communicating and posterior communicating arteries.

In recent years, there has been particular interest in advanced MRI techniques. Arterial spin labelling (ASL) MRI uses radiofrequency pulses to non-invasively label water protons in blood, and can measure vasodilator capacity in symptomatic carotid stenosis. ${ }^{18} \mathrm{~T} 2 *$-based blood oxygen level-dependent contrast (BOLD) MRI uses deoxyhaemoglobin as an indicator of OEF. Cerebrovascular reactivity (CVR) can be quantified using hypercapnia BOLD MRI with good reproducibility. ${ }^{19}$ The technique involves serial MR scans obtained during periods of normocapnia and hypercapnia. CVR maps are then obtained and cerebrovascular vasodilatory capacity can be assessed. An increase in the BOLD signal indicates cerebral vasodilatation, whereas loss of $\mathrm{CO}_{2}$-induced BOLD reactivity suggests maximum compensatory vasodilatation. As BOLD MRI allows whole-brain imaging, it is possible to assess regional variations in CVR.

In spite of the recent developments in advanced MR techniques, PET has been the investigation of choice in major trials evaluating CMP and revascularisation techniques (to be discussed later). There are however other complexities with PET imaging in addition to cost and unavailability. While the original definition of CMP was increased OEF, there has been much heterogeneity in what is considered to be CMP. Some investigators have used a hemispheric OEF ratio of $>1.13$ to define CMP (Carotid Occlusion Surgery Study (COSS) study), while others have included decreased $\mathrm{CBF}$ and decreased $\mathrm{CBF}$ / $\mathrm{CBV}$ ratio in addition to increased OEF. ${ }^{20}$

\section{PROGNOSIS AND OUTCOMES}

Flaherty et al reported the risk of stroke in patients with symptomatic carotid occlusion to be $10 \%$ and $14 \%$, at 1 and 5 years, respectively. The same study also reported a 
mortality of $29 \%$ at 5 years. ${ }^{1}$ The risk of recurrent stroke in patients with symptomatic $\mathrm{CAO}$ is $2.4 \%-7 \%$ per year, and this rises significantly in the presence of haemodynamic impairment due to exhausted cerebrovascular reserve. ${ }^{821-23}$ The ipsilateral ischaemic and all stroke risk was found to be higher in patients with CMP $(17.1 \%$ and $26 \%$, respectively), than those without CMP $(3.1 \%$ and $9.2 \%$, respectively).$^{20}$ The stroke risk in patients without CMP decreased over time; however, there was no such reduction in stroke risk over time in patients with CMP. The presence of CMP and bilateral asymptomatic lacunar infarcts were significant independent predictors of future ipsilateral ischaemic strokes. The study included patients with middle cerebral and internal carotid artery occlusive disease, and a separate analysis of the two groups revealed that CMP was a predictor of recurrent ischaemic stroke in both. ${ }^{20}$

The ability of patients with CAO and CMP to lead normal lives may be impaired in other ways, separate from the devastating effect of stroke. There is now accumulating evidence of impaired cognition and quality of life in patients with CAO. ${ }^{24}{ }^{25}$ What is not known is the long-term effects of CAO and CMP on mood, level of function and disability. There is a need for high-quality research evaluating these specific domains in relation to revascularisation for patients with $\mathrm{CAO}$.

\section{MANAGEMENT}

\section{Revascularisation}

An important first step is to distinguish between complete occlusion and near-occlusion. Symptomatic internal carotid artery near-occlusion presents a strong case for revascularisation through carotid endarterectomy (CEA) or carotid artery stenting (CAS), in order to reduce future stroke risk. While CAS in patients with near-occlusion can be effective in preventing stroke recurrence, there have been concerns with cerebral hyperperfusion syndrome (CHS) and postprocedure vascular events. ${ }^{26}$ There is of course a risk of CHS with any carotid revascularisation procedure, and this was in fact originally described after CEA. In older people, CAS is associated with increased stroke risk, whereas CEA has similar neurological outcomes in both the young and old but at the expense of increased mortality in the elderly. ${ }^{27}$

The evidence for revascularisation in the setting of complete occlusion is weak. Yasargil was the first to describe an anastomosis between superficial temporal artery and a branch of the middle cerebral artery (figure 3$).^{28}$ While initially incorporated into clinical practice, bypass surgery was abandoned after publication of the initial extracranial-intracranial (EC-IC) bypass surgery, which showed no benefit with surgery over best medical treatment. ${ }^{29}$ The study was however criticised for poor design and implementation. Additionally, it was hypothesised that surgery should only be offered to patients with haemodynamic impairment. ${ }^{22}$

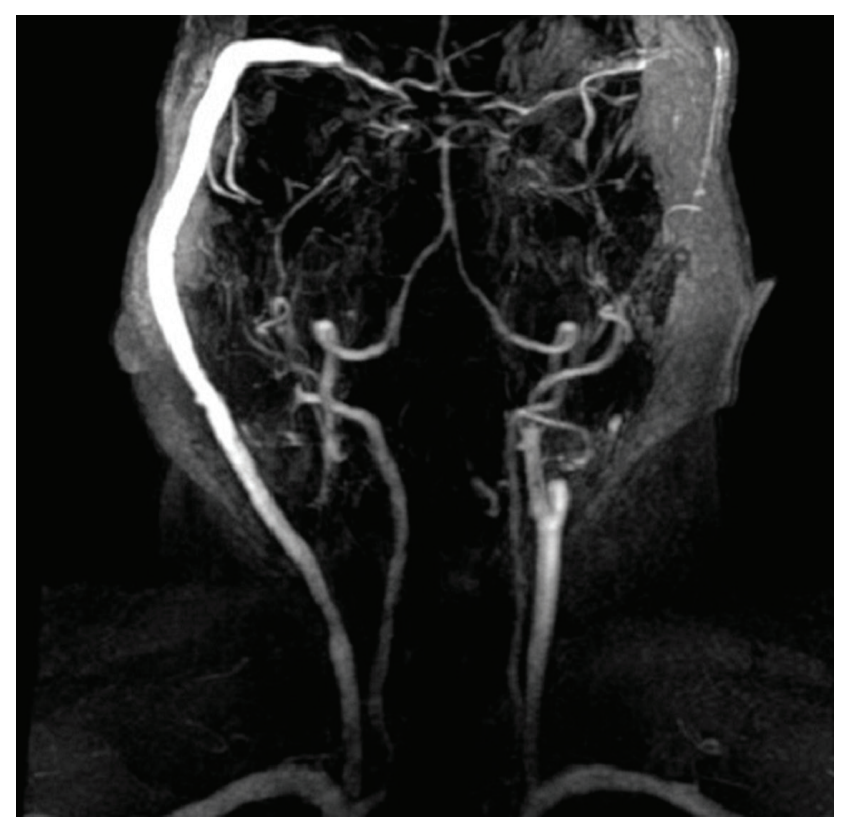

Figure 3 Patient with bilateral carotid occlusive disease who has had a right extracranial-intracranial bypass shunt surgery.

The COSS and Japanese EC-IC Bypass Trial (JET) were set up to answer this question. JET showed benefit at 2 years (second interim analysis) for patients undergoing EC-IC bypass, but the final results were never published. ${ }^{30}$ COSS was stopped early after an interim analysis showed no additional benefit with surgery compared with best medical treatment. ${ }^{31}$ Both studies had recruitment problems, and required PET imaging, which is expensive and not readily available. A subsidiary study of COSS also found that EC-IC bypass surgery did not improve cognitive function in patients with increased OEF. ${ }^{32}$ The study however was small, and did not evaluate quality of life, level of disability or mood. Nevertheless, anecdotal evidence suggests that EC-IC surgery may be of benefit in carefully selected patients with $\mathrm{CAO}$ and haemodynamic impairment benefit from revascularisation. Research evaluating the natural history of patients with CAO is needed.

Finally, another way to revascularise patients with CAO is through CAS, which has been shown to be feasible and practical; ${ }^{33}$ however, larger trials are needed to prove efficacy and long-term follow-up data.

\section{MEDICAL MANAGEMENT}

Conventional vascular risk factors should be addressed and appropriately treated. The benefit of strict blood pressure (BP) control in CAO and CMP is controversial. The loss of compensatory cerebrovascular vasodilatory capacity and the resultant uncoupling of cerebral autoregulation from systemic BP makes cerebral perfusion susceptible to any fall in systemic BP, especially in borderzone regions. Aggressive BP lowering in this context may therefore lead to unwanted clinical outcomes. 
The presence of CMP seems to modify the relationship between BP and stroke risk. Patients with lower BP and CMP had a higher stroke risk, while those with a higher BP without CMP had a greater stroke risk. ${ }^{35}$ In the non-surgical control arm of COSS, lowering BP $(\leq 130 / 85)$ was associated with lower subsequent stroke risk. ${ }^{36}$ On the other hand in another study, lowering BP ( $<130$ systolic) was noted to increase the risk of ipsilateral stroke in those with CMP ${ }^{37}$ Guidelines in the UK recommend a BP target of 130-150 systolic for those with 'severe bilateral carotid stenosis'. ${ }^{38}$ Although CAO and severe bilateral carotid stenosis are not the same entities, a proportion of patients with the latter would have developed CMP.

\section{OUR RECOMIMENDATIONS}

While we recommend liberal BP control (systolic 130-150) to avoid cerebral hypoperfusion, it is important to tailor the target BP for an individual patient according to clinical response. Particular consideration should be given to the frequency and severity of symptoms that occur with lowering BP, presence of postural hypotension and the severity of haemodynamic impairment found on CBF studies. Any depletion of intravascular volume should be corrected, and the temporary cessation of antihypertensives should be considered at times of hypovolaemia or expected hypovolaemia (such as during periods of fasting), or when on holiday in warm countries (case report).$^{39}$

Finally, notwithstanding the unestablished benefit from surgical procedures to improve $\mathrm{CBF}$, it is the opinion of the authors that certain patients may still benefit from revascularisation. Such patients may include those who are thought to be developing cognitive impairment related to $\mathrm{CAO}$, with proven CMP from haemodynamic studies, and those who are relatively young with good baseline functional status, favourable anaesthetic risk profile and no life-limiting comorbidities.

\section{CONCLUSIONS}

Patients with carotid occlusive disease and CMP may present to multiple specialities with a wide-ranging clinical phenotype, and should be considered in those with watershed infarcts, collapses and TIAs or episodic movements associated with an orthostatic component. Early identification of patients with CMP may allow appropriate management and focus on strategies which may maintain or improve $\mathrm{CBF}$, while avoiding potentially harmful treatment.

Contributors SM was responsible for the conceptualisation of the work. The literature review was performed by MM and SM. The initial draft was prepared by MM, with critical revisions and additions by NS, PMB and SM. All authors have approved the final version of the manuscript.

Funding This research received no specific grant from any funding agency in the public, commercial or not-for-profit sectors.

Competing interests None declared.

Provenance and peer review Not commissioned; externally peer reviewed.

Open Access This is an Open Access article distributed in accordance with the Creative Commons Attribution Non Commercial (CC BY-NC 4.0) license, which permits others to distribute, remix, adapt, build upon this work non-commercially, and license their derivative works on different terms, provided the original work is properly cited and the use is non-commercial. See: http://creativecommons.org/ licenses/by-nc/4.0/

Correction notice This article has been corrected since it published Online First. Figures 1, 2 and 3 have been corrected as well as the key points.

(c) Article author(s) (or their employer(s) unless otherwise stated in the text of the article) 2017. All rights reserved. No commercial use is permitted unless otherwise expressly granted.

\section{REFERENCES}

1. Flaherty ML, Flemming KD, McClelland R, et al. Population-based study of symptomatic internal carotid artery occlusion: incidence and long-term follow-up. Stroke 2004;35:e349-e352.

2. van Laar PJ, Hendrikse J, Klijn CJ, et al. Symptomatic carotid artery occlusion: flow territories of Major brain-feeding arteries. Radiology 2007;242:526-34.

3. Krapf H, Widder B, Skalej M. Small rosary-like infarctions in the centrum ovale suggest hemodynamic failure. Am J Neurorad 1998;19:1479-84.

4. Persoon S, Kappelle LJ, Klijn CJ. Limb-shaking transient ischaemic attacks in patients with internal carotid artery occlusion: a casecontrol study. Brain 2010;133(Pt 3):915-22.

5. Kashiwazaki D, Kuroda S, Terasaka S, et al. Carotid occlusive disease presenting with loss of consciousness. No Shinkei Geka 2005;33:29-34.

6. Welsh T, Maddula M, Hickey P, et al. Cerebral misery perfusion presenting with transient global amnesia - implications for pathogenesis. Int J Stroke 2012;7(suppl 2):1-79.

7. Garrett MC, Komotar RJ, Starke RM, et al. Radiographic and clinical predictors of hemodynamic insufficiency in patients with atheroocclusive disease. J Stroke Cerebrovasc Dis 2008;17:340-3.

8. Persoon S, Luitse MJ, de Borst GJ, et al. Symptomatic internal carotid artery occlusion: a long-term follow-up study. J Neurol Neurosurg Psychiatry 2011;82:521-6.

9. AbuRahma AF, Pollack JA, Robinson PA, et al. The reliability of color duplex ultrasound in diagnosing total carotid artery occlusion. Am J Surg 1997;174:185-7.

10. Ohm C, Bendick PJ, Monash J, et al. Diagnosis of total internal carotid occlusions with duplex ultrasound and ultrasound contrast. Vasc Endovascular Surg 2005;39:237-43.

11. Chen CJ, Lee TH, Hsu HL, et al. Multi-Slice CT angiography in diagnosing total versus near occlusions of the internal carotid artery: comparison with catheter angiography. Stroke 2004;35:83-5.

12. Herzig R, Burval S, Krupka B, et al. Comparison of ultrasonography, $\mathrm{CT}$ angiography, and digital subtraction angiography in severe carotid stenoses. Eur J Neurol 2004;11:774-81.

13. El-Saden SM, Grant EG, Hathout GM, et al. Imaging of the internal carotid artery: the dilemma of total versus near total occlusion. Radiology 2001;221:301-8.

14. Carlson AP, Yonas H, Chang YF, et al. Failure of cerebral hemodynamic selection in general or of specific positron emission tomography methodology?: carotid occlusion surgery study (COSS). Stroke 2011;42:3637-9.

15. Yonas H, Good WF, Gur D, et al. Mapping cerebral blood flow by xenon-enhanced computed tomography: clinical experience. Radiology 1984;152:435-42.

16. Hasegawa $Y$, Yamaguchi T, Tsuchiya T, et al. Sequential change of hemodynamic reserve in patients with Major cerebral artery occlusion or severe Stenosis. Neuroradiology 1992;34:15-21.

17. Yokota C, Hasegawa Y, Minematsu K, et al. Effect of acetazolamide reactivity on [corrected] long-term outcome in patients with Major cerebral artery occlusive diseases. Stroke 1998;29:640-4.

18. Bokkers RP, van Osch MJ, van der Worp HB, et al. Symptomatic carotid artery Stenosis: impairment of cerebral autoregulation measured at the brain tissue level with arterial spin-labeling MR imaging. Radiology 2010;256:201-8.

19. Goode SD, Krishan S, Alexakis C, et al. Precision of cerebrovascular reactivity assessment with use of different quantification methods for hypercapnia functional MR imaging. AJNR 2009;30:972-7.

20. Yamauchi $\mathrm{H}$, Higashi T, Kagawa S, et al. Is misery perfusion still a predictor of stroke in symptomatic Major cerebral artery disease? Brain 2012;135(Pt 8):2515-26.

21. Hankey GJ, Warlow CP. Prognosis of symptomatic carotid artery occlusion - an overview. Cerebrovasc Dis 1991;1:245-56.

22. Klijn CJ, Kappelle LJ, van Huffelen AC, et al. Recurrent ischemia in symptomatic carotid occlusion: prognostic value of hemodynamic factors. Neurology 2000;55:1806-12. 
23. Grubb RL, Derdeyn CP, Fritsch SM, et al. Importance of hemodynamic factors in the prognosis of symptomatic carotid occlusion. JAMA 1998;280:1055-60.

24. Fiedler J, Priban V, Skoda O, et al. Cognitive outcome after EC-IC bypass surgery in hemodynamic cerebral ischemia. Acta Neurochir 2011;153:1303-12.

25. Bakker FC, Klijn CJ, van der Grond J, et al. Cognition and quality of life in patients with carotid artery occlusion: a follow-up study. Neurology 2004;62:2230-5.

26. Son S, Choi DS, Kim SK, et al. Carotid artery stenting in patients with near occlusion: a single-center experience and comparison with recent studies. Clin Neurol Neurosurg 2013;115:1976-81.

27. Perler BA. Outcomes of carotid endarterectomy and stenting in elderly patients. JAMA 2014;311:1244-5.

28. Yasargil MG. Diagnosis and indications for operations in cerebrovascular occlusive disease, in Microsurgery Applied to Neurosurgery, Stuttgart, Georg Thieme Verlag, 1969:95-119.

29. Failure of extracranial-intracranial arterial bypass to reduce the risk of ischemic stroke. results of an international randomized trial. the EC/ IC bypass Study Group. N Engl J Med 1985;313:1191-200.

30. Japanese EC-IC Bypass Trial (JET Study): The Second Interim analysis. Surg Cereb Stroke 2002;30:434-7.

31. Powers WJ, Clarke WR, Grubb RL, et al. Extracranial-intracranial bypass surgery for stroke prevention in hemodynamic cerebral ischemia: the carotid occlusion surgery study randomized trial. JAMA 2011;306:1983-92.

32. Marshall RS, Festa JR, Cheung YK, et al. Randomized evaluation of carotid occlusion and neurocognition (RECON) trial: main results. Neurology 2014;82:744-51

33. Bhatt $A$, Majid A, Kassab M, et al. Chronic total symptomatic carotid artery occlusion treated successfully with stenting and angioplasty. J Neuroimaging 2009;19:68-71.
34. Shojima M, Nemoto S, Morita A, et al. Protected endovascular revascularization of subacute and chronic total occlusion of the internal carotid artery. AJNR 2010;31:481-6.

35. Yamauchi H, Higashi T, Kagawa S, et al. Impaired perfusion modifies the relationship between blood pressure and stroke risk in Major cerebral artery disease. J Neurol Neurosurg Psychiatry 2013;84:1226-32.

36. Powers WJ, Clarke WR, Grubb RL, et al. Lower stroke risk with lower blood pressure in hemodynamic cerebral ischemia. Neurology 2014;82:1027-32.

37. Yamauchi H, Kagawa S, Kishibe Y, et al. Misery perfusion, blood pressure control, and 5-year stroke risk in symptomatic Major cerebral artery disease. Stroke 2015;46:265-8.

38. National clinical guidelines for Stroke. Fourth edition: Royal College of Physicians, 2012.

39. Maddula MP, Keegan BC. Limb shaking as a manifestation of Low-flow transient ischemic attacks. Int J Gerontol 2010;4:47-50.

40. Gordon AL, Goode S, D'Souza O, et al. Cerebral misery perfusion diagnosed using hypercapnic blood-oxygenation-level-dependent contrast functional magnetic resonance imaging: a case report. $J$ Med Case Rep 2010;4:54.

41. Furlan AJ, Whisnant JP, Kearns TP. Unilateral visual loss in bright light. an unusual symptom of carotid artery occlusive disease. Arch Neurol 1979;36:675-6.

42. Russell RW, Page NG. Critical perfusion of brain and retina. Brain 1983;106:419-34.

43. Bakker FC, Klijn CJ, Jennekens-Schinkel A, et al. Cognitive disorders in patients with occlusive disease of the carotid artery: a systematic review of the literature. $J$ Neurol 2000;247:669-76. 